\title{
Influence of Artery Straightening on Local Hemodynamics in Left Anterior Descending (LAD) Artery after Stent Implantation
}

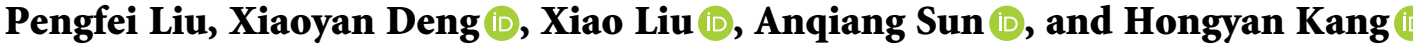 \\ Key Laboratory for Biomechanics and Mechanobiology of the Ministry of Education, \\ School of Biological Science and Medical Engineering, Beijing Advanced Innovation Center for Biomedical Engineering, \\ Beihang University, Beijing 100083, China \\ Correspondence should be addressed to Anqiang Sun; saq@buaa.edu.cn and Hongyan Kang; hongyankang@buaa.edu.cn
}

Received 5 June 2019; Accepted 20 April 2020; Published 22 May 2020

Academic Editor: Mariantonietta Cicoira

Copyright (c) 2020 Pengfei Liu et al. This is an open access article distributed under the Creative Commons Attribution License, which permits unrestricted use, distribution, and reproduction in any medium, provided the original work is properly cited.

Objectives. The study investigates local hemodynamic environment changes caused by straightening phenomenon and the relationship between straightening phenomenon and in-stent restenosis. Background. Intravascular intervention is an effective treatment in restoring the normal flow conditions and vascular lumen. Unfortunately, in-stent restenosis often occurs in a subset of patients after stent implantation and limits the success of stent implantation outcomes. The implanted stent usually causes artery straightening locally, rather than coinciding and adjusting to the physiological curve exactly. Artery straightening would apparently modify the artery geometry and therefore alter the local hemodynamic environment, which may result in intimal hyperplasia and restenosis after stenting implantation. Methods. In the current investigation, we verify the hypothesis that the artery straightening influences the local hemodynamic state using the different 3D CT models. Flow analysis for blood in the left anterior descending coronary artery and the straightening model is simulated numerically. Result. The current results reveal that the straightening phenomenon alters the distribution of wall shear stress and flow patterns, decreases the wall shear stress (WSS), and increases the oscillatory shear index (OSI) and the relative residence time (RRT), especially at the proximal and distal areas of stenting. Conclusions. The local straightened geometry established after stent implantation was likely to generate portions of the stenting area to a high risk of neointimal hyperplasia and subsequent restenosis.

\section{Introduction}

Stents are usually implanted into stenotic coronary arteries to improve or restore blood flow environment. However, restenosis has some persistent problems that limit the development of percutaneous coronary intervention $[1,2,3]$. Studies reported that restenosis occurs in $12 \%$ of patients after stent implantation [3].

The mechanisms responsible for restenosis were not yet fully elucidated. Stent geometry and its subsequent effects on localized hemodynamics may cause restenosis [4-6]. Williams et al. reported that reduced vessel compliance and altered distributions of the wall shear stress (WSS) within the stented region could induce restenosis occurrence [7-9]. Besides, previous studies demonstrated that smooth muscle and endothelial cell damage would be implicated as potential factors for stimulating neointimal hyperplasia $[8,10,11]$. There was a putative link between the hemodynamic environment changes and restenosis [12, 13]. Moreover, Regar et al. found that the procedure-specific factors such as implantation technique also can influence restenosis [14].

In addition to these reasons, the existing researches suggested that the straightening phenomenon caused by stent implantation induced changes in local hemodynamic environment and the rate of restenosis occurrence rate [15-18]. However, these studies were just conducted with simple two-dimensional models or three-dimensional idealized models, which cannot illustrate the realistic blood flow environment $[7,19,20]$.

The straightening phenomenon is usually due to the different curvatures between stent and lesion vessels, 
resulting in two evident angle changes near both ends of the stented region. The present study hypothesized that the straightening phenomenon can change the local hemodynamic state and vascular geometry. These changes can increase the possibility of restenosis occurrence and affect the stent surgeries.

In order to verify this hypothesis, the study reconstructed the left anterior descending coronary artery (LAD) and the straightening model based on the realistic computed tomography (CT) images and stent. The normal blow flow condition is used to simulate the local hemodynamic state. This study revealed the changes in local hemodynamics caused by the straightening phenomenon and discussed the relationship between artery straightening and restenosis. The implications of this study help the determination of potential causes of restenosis after stent implantation and the optimization design of the stent.

\section{Materials and Methods}

2.1. Reconstruction of Normal and the Straightening Models. The study reconstructed the realistic coronary artery model based on the CT scan images with Mimics (v15.0, Materialise, Ann Arbor, MI, USA). The CT relevant parameters were described as follows: $0.9 \mathrm{~mm}$ slice thickness, $0.45 \mathrm{~mm}$ slice increment, $0.324 \mathrm{~mm}$ pixel size, a $512 \times 512$ image resolution, and total 293 [21]. The LAD model was obtained from the coronary artery model by using intercept function. The features of stent structure would be neglected especially after the endothelialization [10, 20]. Therefore, only the artery curve information was considered in the modeling process. The straightening model was obtained from LAD fitting with the stent model. Subsequently, simple smoothing and surfacing processes were applied to the models with Geomagic Studio 2012 (3D Systems, Morrisville, NC, USA). The diameter of LAD is $1.7 \mathrm{~mm}$. Using a stent-to-artery diameter ratio of 1.2 , the diameter of the stent was defined as $2 \mathrm{~mm}$, and the length of the stent was $7 \mathrm{~mm}$. The two models are shown in Figure 1.

2.2. Meshing. ANSYS ICEM 16.0 (ANSYS, Inc., Canonsburg, PA, USA) software was used to complete the models meshing. Hexahedral elements were used, and the number of boundary layer was set to 10, with the height ratio 1.1 and initial height $0.009 \mathrm{~mm}$. The total node number of the normal model was 116081 . The total node number of the straightening model was 112476 . Two models' meshes are shown in Figure 2.

2.3. Simulation and Boundary Conditions. Simulation calculation was conducted by ANSYS Fluent 16.0 software (ANSYS, Inc., Canonsburg, PA, USA) under the pulsatile flow conditions. Blood was modeled as a Newtonian fluid and assumed to be homogeneous and incompressible $[22,23]$. The convergence criterion was set to 0.00001 . The vascular wall was postulated to be nonslip of the rigid wall. The inlet condition used the realistic blood flow velocity (Figure 3) [24]. The numerical simulation was conducted based on a three-dimensional incompressible Navier-Stokes equation and the conservation of mass:

$$
\begin{array}{r}
\rho\left[\frac{\partial \mathbf{u}}{\partial t}+(\mathbf{u} \cdot \nabla) \mathbf{u}\right]+\nabla p-\mu \nabla^{2} \mathbf{u}=0 \\
\nabla \cdot \mathbf{u}=0
\end{array}
$$

where $\mathbf{u}$ and $p$ represent the fluid velocity vector and pressure, respectively. $\rho$ and $\mu$ are the density and viscosity of blood $\left(\mu=3.5 \times 10^{-3} \mathrm{~kg} / \mathrm{m} \cdot \mathrm{s}\right.$ and $\left.\rho=1050 \mathrm{~kg} / \mathrm{m}^{3}\right)$ [25]. SIMPLE algorithm was used to calculate the blood flow velocity, and pressure-based solver was used for pressure correction and to solve the momentum equation. Computation period was set to $1.08 \mathrm{~s}$, each step for $0.008 \mathrm{~s}$, with 136 steps in each cycle. Every cycle was required to obtain a convergence for the transient analysis. The largest number of iterations in every step was 500, and the total steps were 1000. The whole computational process spanned twelve working days.

2.4. Hemodynamic Parameters. Postprocessing was conducted with Matlab (The Math Works, Natick, Mass) and Tecplot 360 2013R1 software using the data exported from ANSYS FLUENT 16.0. Three WSS-based hemodynamic parameters (TAWSS, OSI, and RRT) were calculated based on the research by Claudio Chiastra [13].

Wall shear stress (WSS) was defined to be the product of fluid viscosity and shearing velocity of the neighboring vascular wall. We used $1050 \mathrm{~kg} / \mathrm{m}^{-3}$ as the viscosity of blood. WSS had close connection with blood characters, blood flow velocity, and vascular morphology.

The time-averaged wall shear stress (TAWSS) was the average of WSS in the entire cardiac cycle of WSS. TAWSS was described as the characteristics of WSS in pulsatile flow. TAWSS was calculated as follows:

$$
\text { TAWSS }=\frac{1}{T} \int_{0}^{T}|\mathbf{W S S}(\mathbf{s}, t)| \cdot d t,
$$

where $\mathrm{T}$ is the duration of the cardiac cycle and $\mathbf{s}$ is the position on the vascular wall.

The oscillatory shear stress index (OSI) was the nondimensional parameter; it indicated the magnitude of WSS fluctuations during a cardiac cycle. It is defined as follows:

$$
\text { OSI }=0.5\left[1-\left(\frac{\left|(1 / T) \int_{0}^{T} \operatorname{WSS}(\mathbf{s}, t) \cdot \mathrm{d} t\right|}{(1 / T) \int_{0}^{T}|\mathrm{WSS}(\mathrm{s}, t)| \cdot \mathrm{d} t}\right)\right] .
$$

The high OSI leads to the lack of endometrial cells function and the decisive factor to change cellular structure cyclic stress.

The relative residence time (RRT) is introduced:

$$
\mathrm{RRT}=\frac{1}{(1-2 \cdot \mathrm{OSI}) \cdot \mathrm{TAWSS}}
$$

The RRT is inversely proportional to the magnitude of the TAWSS vector and has obvious connections to the 


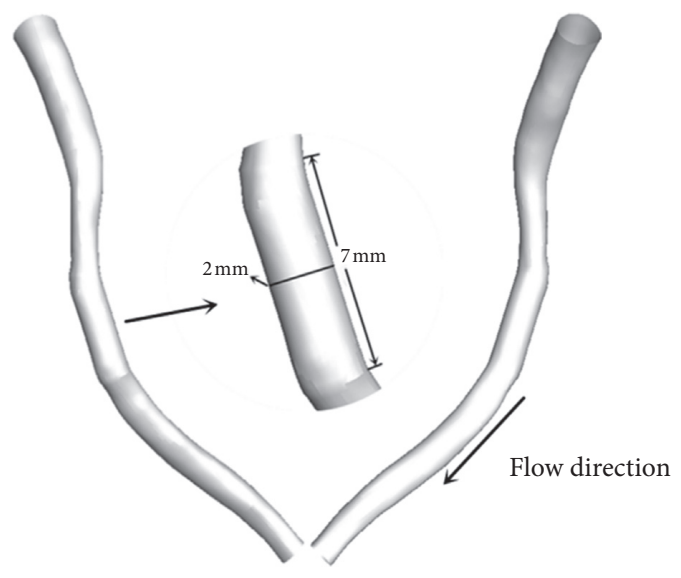

(a)

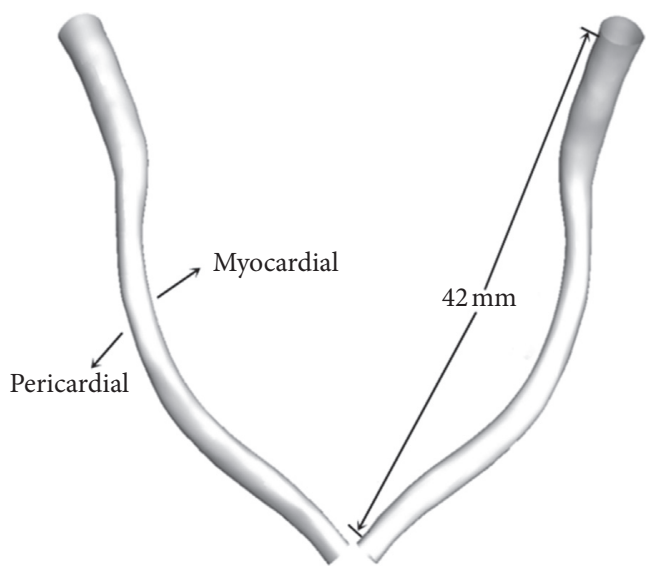

(b)

Figure 1: (a) Straightening and (b) normal models. The enlarged image shows the straightening phenomenon caused by stent implantation.

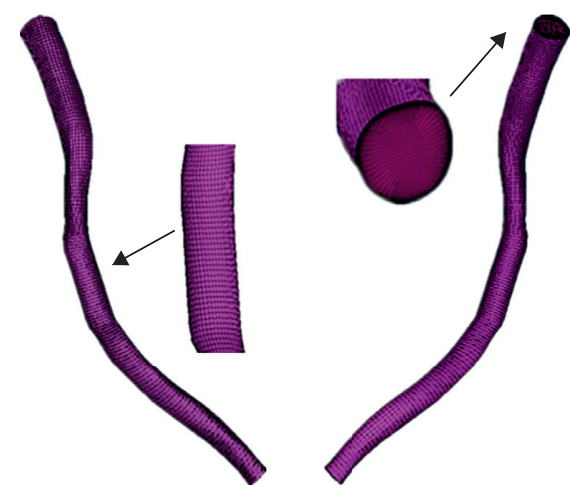

(a)

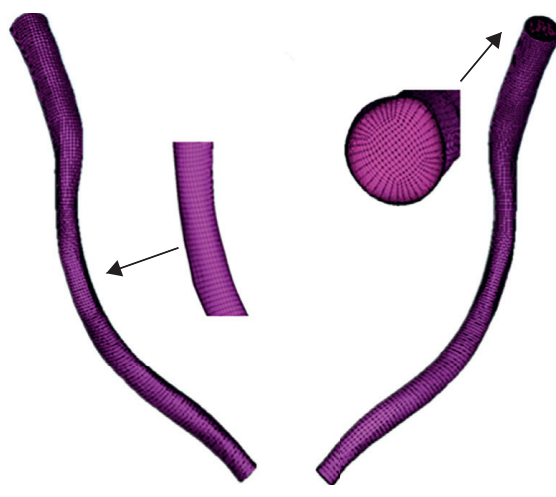

(b)

FIGURE 2: Mesh of the (a) straightening and (b) normal models.

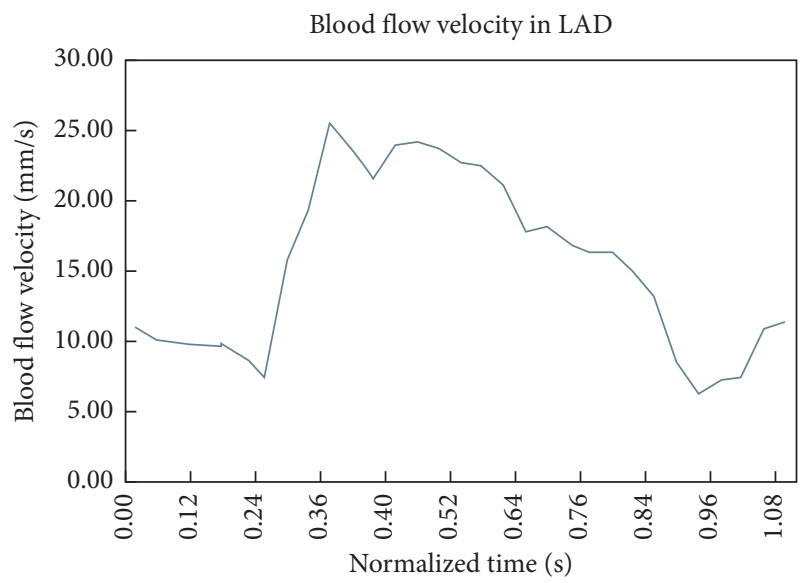

FIGURE 3: Inlet velocity pulsating flow. A representative waveform describing the average blood flow velocity measured in the proximal portion of a human left anterior descending coronary artery during a cardiac cycle. This waveform was used to complete the time-dependent simulations in the present study. biological mechanisms of atherosclerosis. The OSI modifies the TAWSS effects on the RRT at a given region of the endothelium. Therefore, the RRT parameter includes the effects of both OSI and TAWSS.

\section{Results}

Two reconstruction models were used for simulation calculation; the best convergence cycle of seven cycles was selected to analyze the experiment data. On the basis of the hemodynamic parameters (blood flow velocity, WSS, TAWSS, OSI, and RRT), the study aimed at investigating the influence of straightening phenomenon after stent implantation on local hemodynamic environment in LAD.

3.1. The Blood Flow Velocity. Four points from one cardiac cycle are shown in Figure 4; early systole $\left(t_{1}\right)$, peak systole $\left(t_{2}\right)$, second peak systole $\left(t_{3}\right)$, and end systole $\left(t_{4}\right)$ were selected from one cardiac cycle. 


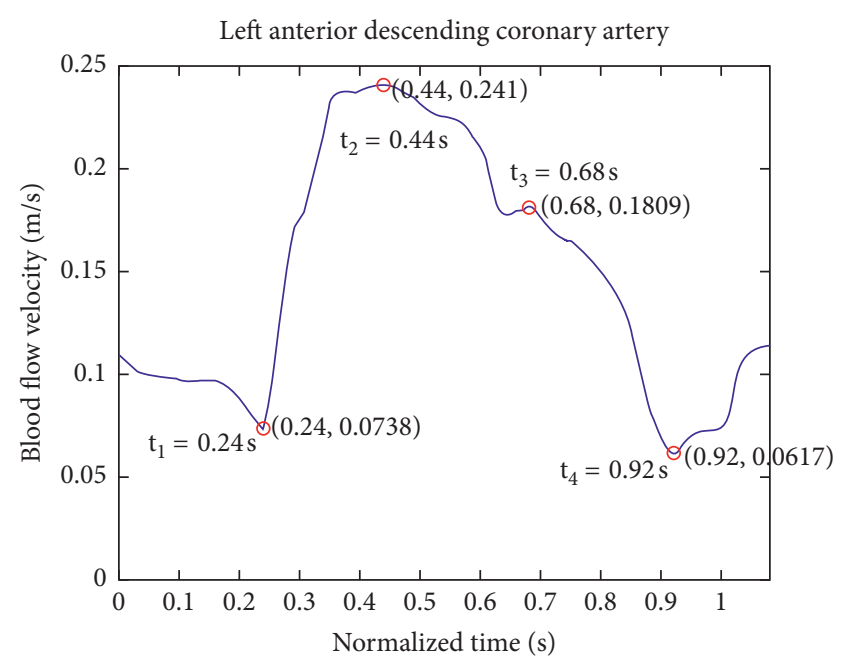

FIGURE 4: Four typical points during one cardiac cycle. $t_{1}, t_{2}, t_{3}$, and $t_{4}$ are selected from one cardiac cycle and, respectively, represent early systole, peak systole, second peak systole, and end systole.

Figure 5 shows the velocity streamlines in normal and straightening models. There was no obvious difference in the flow patterns and velocity between the models except the stenting area. In the stented area, the blood velocity of the straightening model obviously decreased. The highest blood flow velocity and the maximum difference of velocity between models emerged at the $t_{2}$ moment. In contrast, the $t_{4}$ moment had the lowest blood flow velocity and the minimum difference of velocity between models. The flow pattern of the straightening model obviously altered at time $t_{1}$ and time $t_{3}$. According to the velocity contours, the velocity near the pericardial surface was higher. The difference of velocity distribution between two models increased from $t_{1}$ to $t_{3}$, and the maximum disparity occurred at time $t_{3}$.

3.2. WSS and TAWSS. Contours of the WSS at the four points are shown in Figure 6. The distributions of WSS between models except the stented area were similar. In the stented area, the distributions of WSS were significantly altered, and the WSS was reduced by the straightening phenomenon. The WSS of the straightening model illustrates a gradient increase along the pericardial surface. To compare with other points, the maximum change in WSS between two models occurred at the $t_{2}$ moment, and the minimum difference occurred at time $t_{4}$. From the straightening model, the highest average WSS was $8.77 \mathrm{~Pa}$ at time $t_{2}$, and the lowest average WSS was $2.20 \mathrm{~Pa}$ at time $t_{4}$. Meanwhile, from the normal model, it increased to $11.27 \mathrm{~Pa}$ at time $t_{2}$ and $2.68 \mathrm{~Pa}$ at time $t_{4}$.

The distribution of TAWSS is described in Figure 7 (TAWSS counters on different models), and similar distribution of TAWSS was observed compared with abovedescribed WSS distribution. Within the stented area, the TAWSS of the straightening model significantly decreased, especially at the inlet and outlet of the stenting region and the pericardial surface. Examination of TAWSS as a function of normalized axial length revealed that TAWSS of the straightening model was greater at the pericardial compared to the myocardial luminal surface. The largest TAWSS difference occurred at the inlet area.

3.3. OSI and RRT. Figure 8 shows the contours of OSI on the different models. Generally, the areas of high OSI were observed in areas of low WSS where the direction changed frequently. The significantly higher OSI of the straightening model was observed at the inlet of the stented area compared to the normal (shown in enlarge image).

The contours of RRT under different conditions are shown in Figure 9. The straightening phenomenon increased RRT values within the stented area, especially the RRT at the inlet of the stent and the pericardial surface. From the straightening model, the difference of RRT on both sides was higher. The highest RRT of the straightening model was calculated at the inlet of the stent along the myocardial surface, and the maximum RRT difference between two models occurred in this region. Beyond all that, the straightening phenomenon had a serious influence on the distribution of RRT.

\section{Discussion}

Stenting is used as an important treatment for critical artery stenosis. However, postoperation complications are still perplexing the patients over time, for example, neointimal hyperplasia and subsequent restenosis $[7,8,9,17,26]$. The reasons causing the restenosis are still being explored. Numerous studies have been conducted to investigate the potential influences including stent types, carrying drugs, and many postoperative and intraoperative factors [26-28]. As the compliance of the stent usually does not adjust to the curved artery, artery "straightening" is easily formed at the stented areas, potentially changing the local blood flow features and consequently influencing arterial cells behaviors and inducing tissue remodeling. It would be very useful and necessary to investigate the detailed hemodynamic changes in the straightened areas after stenting. Some studies focused on the straightening of vascular caused by stent implantation, but the relationship between the straightening phenomenon and restenosis was unclear $[15-17,28]$. In these published studies, idealized cylindrical models were usually used to examine flow patterns through stented vessels with "straightening" $[7,19,20]$. However, the detailed local hemodynamic features changed by straightening phenomenon in realistic $3 \mathrm{D}$ models was not clear.

In the last 5 decades, the low wall shear stress hypothesis of atherosclerosis proposed by Caro et al. has been validated [29]. The connection between low WSS and high intimal proliferation had been further certified in rodents [30]. The vascular regions subjected to WSS below $0.5 \mathrm{~Pa}$ have been shown to strongly correlate with sites of intimal thickening $[31,32]$. Low TAWSS was thought to be associated with regions of cellular proliferation and the potential factors for the development of neointimal.[33]. The high OSI created greater endothelial cells proliferation, and increased RRT 


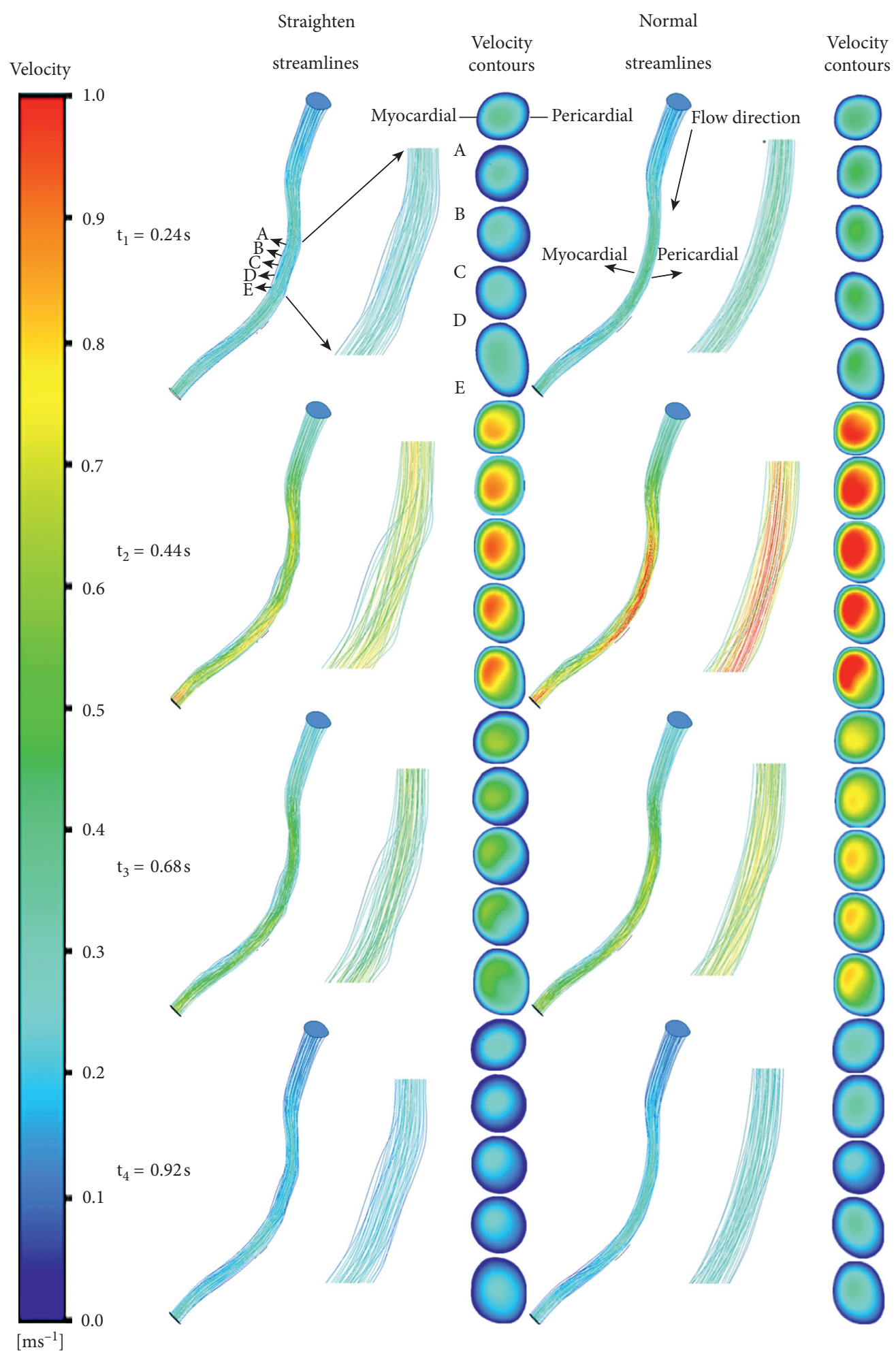

FIGURE 5: The streamlines near the left anterior descending coronary artery at four typical points with the various models. Cross sections are velocity contours in different moments of the different models. A, B, C, D, and E are selected from the models, and the first point is located at the inlet of the stented region. The distance between two points is $1 \mathrm{~mm}$, and these points are also shown. Slicing in these points along the direction perpendicular to the centerline forms the cross sections. 


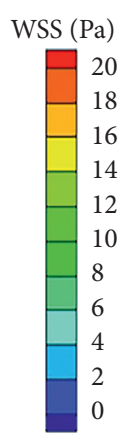

WSS (Pa)

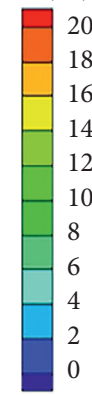

WSS $(\mathrm{Pa})$
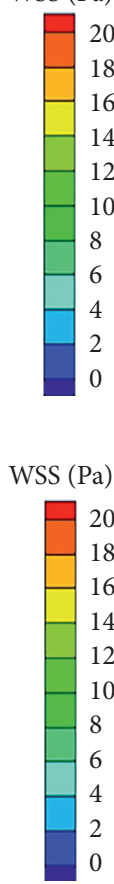

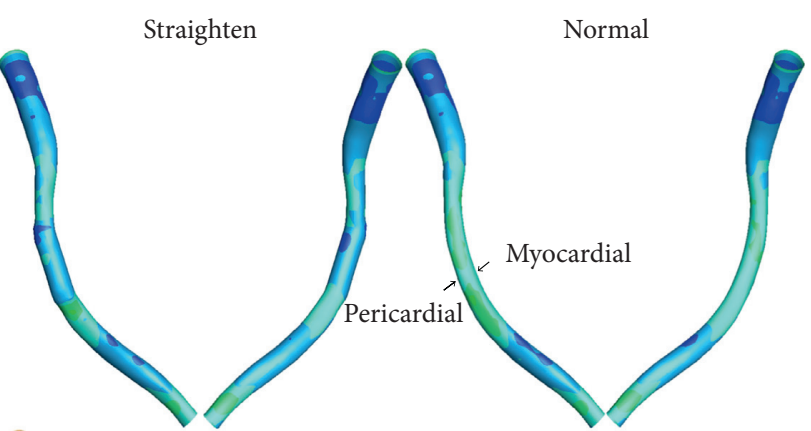

(a)

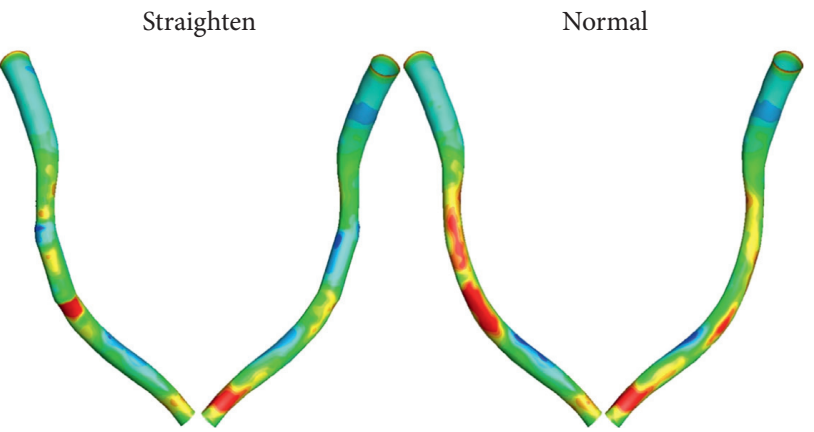

(b)

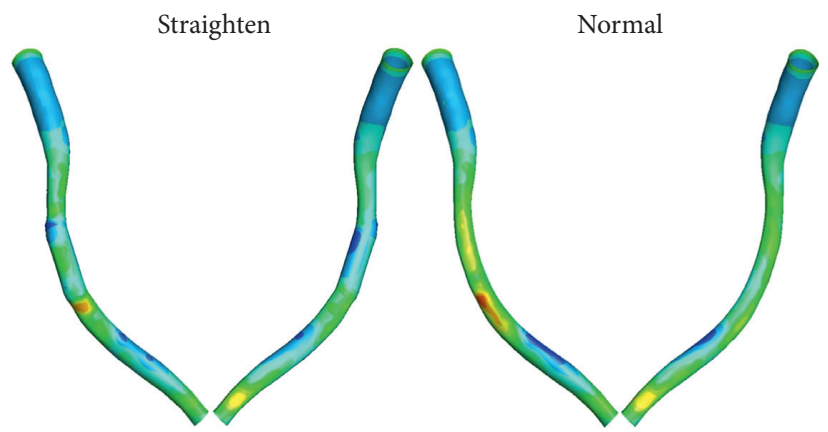

(c)

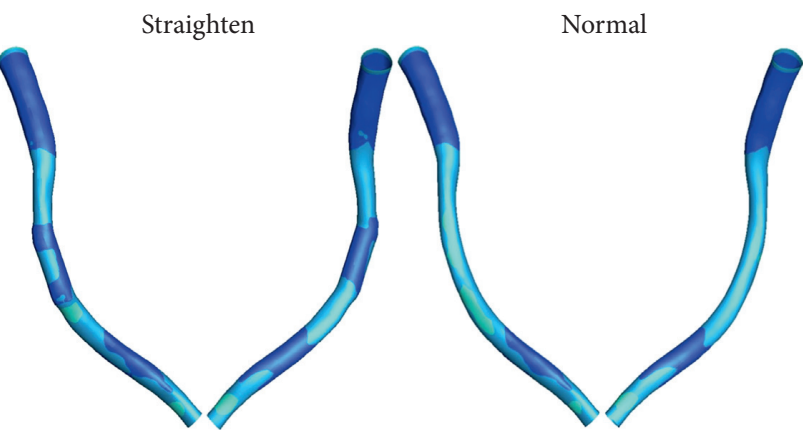

(d)

Figure 6: Counters of WSS at four typical points: (a) $t_{1}=0.24 \mathrm{~s}$; (b) $t_{2}=0.44 \mathrm{~s}$; (c) $t_{3}=0.68 \mathrm{~s}$; (d) $t_{4}=0.92 \mathrm{~s}$.

had obvious connections with biological mechanisms of atherosclerosis [18]. Moreover, Colombo pointed that abnormal blood flow patterns can promote inflammation, endothelial cells proliferation, and thrombosis [34].

In study, the straightening phenomenon caused by stent implantation was an potential factor to the restenosis. Normal and straightening models based on CT images were reconstructed, and blood flow parameters in LAD were used as boundary conditions. The numerical simulation results revealed the straightening phenomenon altered the lumen geometry and consequently changed blood flow patterns in the local areas. The velocity and WSS in the stented area decreased after the straightening phenomenon. Besides, the RRT and OSI increased on the straightening model. The significant changes 


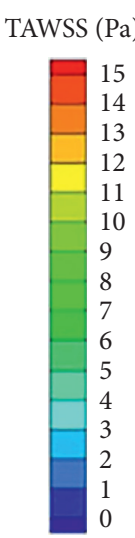

\begin{tabular}{|l|}
\hline 15 \\
\hline 14 \\
\hline 13 \\
12 \\
\hline 11 \\
10 \\
9 \\
8 \\
7 \\
6 \\
5 \\
4 \\
3 \\
2 \\
1 \\
0
\end{tabular}

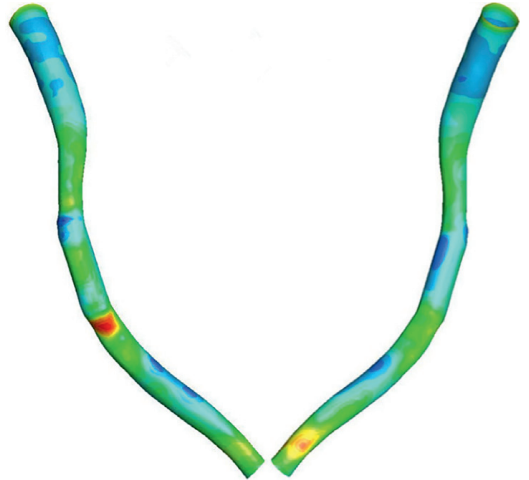

(a)

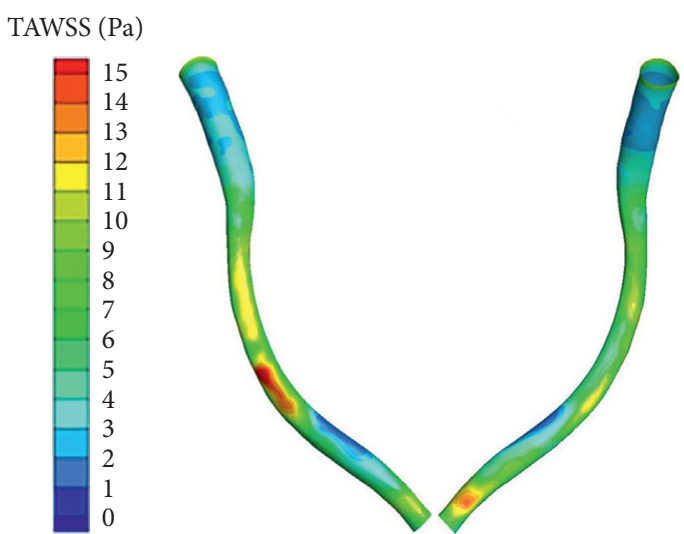

(b)

FIGURE 7: Counters of TAWSS: (a) straightening; (b) normal.

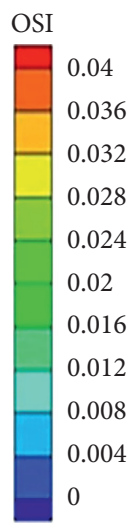

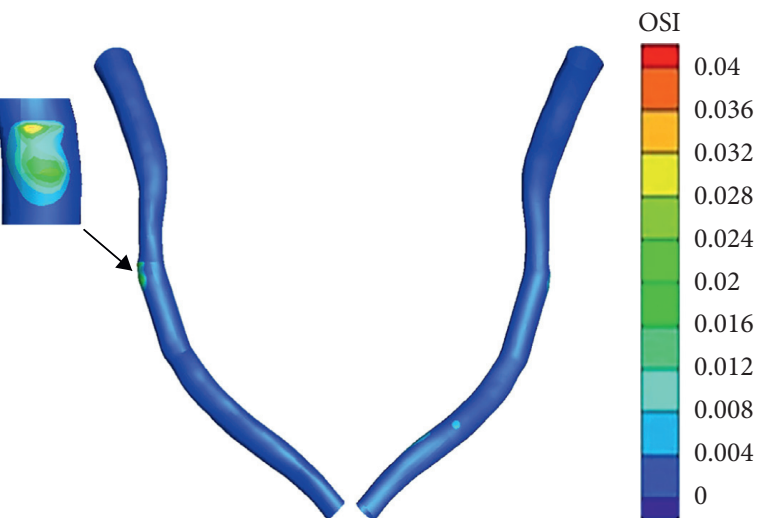

(a)

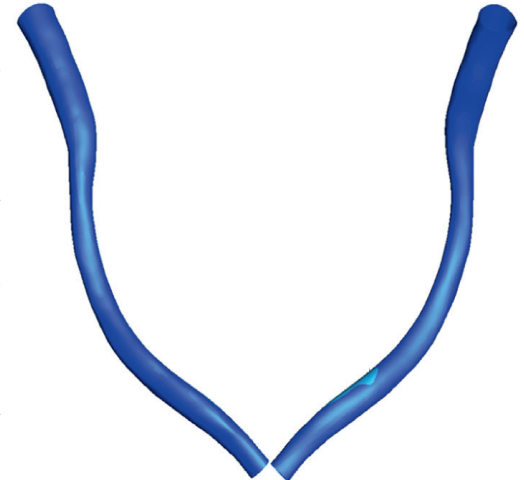

(b)

FIgURE 8: Counters of OSI: (a) straightening; (b) normal.

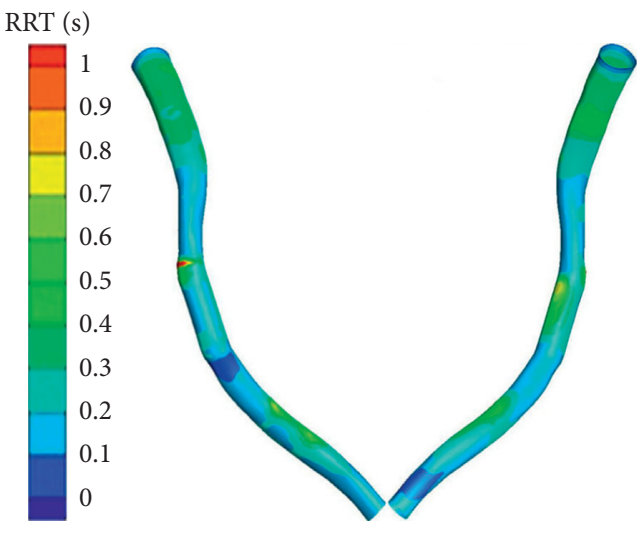

(a)
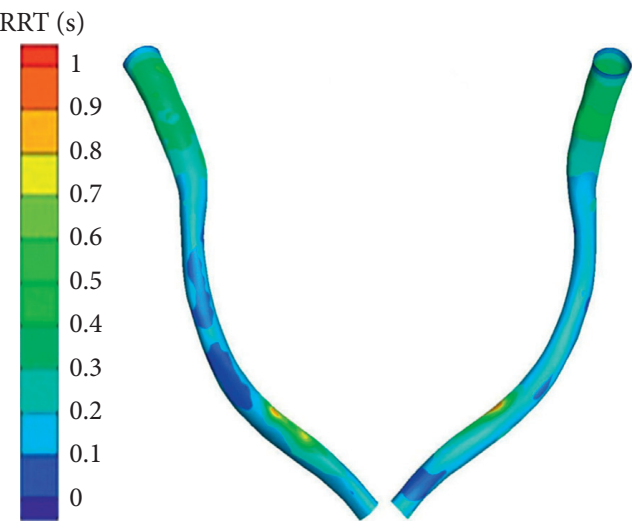

(b)

FIGURE 9: Counters of relative residence time (RRT): (a) straightening; (b) normal.

in parameters happened especially at the proximal and distal end of stented areas and the pericardial surface. As the low WSSs, high RRTs, and high OSIs were all indexes of restenosis subsequently [18], the straightening phenomenon hereby would induce a hemodynamic environment in LAD that promotes these conditions. Therefore, we conclude that the straightening phenomenon could affect the hemodynamic environment in the LAD and expose the endothelial cells to low WSS, high OSI, and high RRT conditions. These can make stented area more likely to cause the restenosis and neointimal hyperplasia, especially at the proximal and distal ends of the stented area and the pericardial surface. This conclusion 
coincided with the findings of Wentzel and colleagues who observed clinical evidence of restenosis in [16].

Because some assumptions and simplification have been used in this study (e.g., the rigid wall assumption and neglect of detailed stent strut features), the results may not perfectly describe the hemodynamic environment in the straightened areas. However, the present study can still provide some clues to investigate the physiological and pathological impacts of straightening phenomenon after stent implantation.

In the future, at least two issues should be highly concerned. The first one is to analyze the artery wall stress and strain in the straightened areas. Because the arterial wall remodeling could be significantly regulated by the stress and strain in the artery [35]. The second one is the drug delivery and deposition characteristics in the special hemodynamic environment caused by straightening phenomenon. Drug eluting stent (DES) is now being prevalently used in clinics, and previous studies had proved the high correlation between local blood flow patterns and drug delivery $[12,36,37]$. Thus, investigating the drug delivery and deposition characteristics in straightened areas will be important in optimizing DES stenting.

\section{Conclusion}

This study investigated the local hemodynamic environment changes caused by straightening phenomenon. And we explored the relationship between the straightening phenomenon and the restenosis. The straightening phenomenon would lead to the decrease in WSS, TAWSS, and blood flow velocity and increase in RRT and OSI. This study concluded that the regional geometry of straightening phenomenon established after stent implantation is likely to lead to portions of the stenting area to a high risk of neointimal hyperplasia and subsequent restenosis (especially in the inlet and outlet of the stent and the pericardial surface). This study could help elucidate the mechanism of restenosis and promote the development of stent technology. This study can also serve as a reminder of the potential risks and provide useful guides in the stent deployment procedures.

\section{Abbreviations}

CT: $\quad$ Computed tomography

LAD: Left anterior descending

WSS: Wall shear stress

TAWSS: Time-averaged wall shear stress

OSI: Oscillatory shear stress index

RRT: Relative residence time.

\section{Data Availability}

The data used to support the findings of this study are included within the article.

\section{Conflicts of Interest}

The authors declare that they have no conflicts of interest.

\section{Acknowledgments}

The authors gratefully acknowledge the generous assistance and valuable advice provided by Professor Fang $\mathrm{Pu}$ and modeling assistance from Minzhen Fan along with simulation assistance provided by Peng Zhang. This work was supported by the National KeyResearch and Development Program of China (2017YFB0702501), the National Natural Science Foundation of China (11872096, 31870940, 11472031, and 11421202), the"111" Project ( B13003 ), and the Natural Science Foundation of Jiangsu Province, China (BK20161366).

\section{References}

[1] J. F. Ladisa, I. Guler, L. E Olson et al., “Three-dimensional computational fluid dynamics modeling of alterations in coronary wall shear stress produced by stent implantation," Annals of Biomedical Engineering, vol. 31, no. 31, pp. 972-980, 2003.

[2] S. B. Simon, "A randomized comparison of coronary-stent placement and balloon angioplasty in the treatment of coronary artery disease. Stent Restenosis Study Investigators," New England Journal of Medicine, vol. 331, no. 8, pp. 496-501, 1994.

[3] K. Palialexis, S. Spiliopoulos, A. Dalakidis et al., "Covered stents for symptomatic iliac artery in-stent restenosis treatment: midterm Results," Hellenic Journal of Radiology, vol. 2, no. 2, 2017.

[4] A. B. Fontaine, D. G. Spigos, G. Eaton et al., "Stent-induced intimal hyperplasia: are there fundamental differences between flexible and rigid stent designs?" Journal of Vascular and Interventional Radiology, vol. 5, no. 5, pp. 739-744, 1994.

[5] A. Kastrati, J. Mehilli, J. Dirschinger et al., "Restenosis after coronary placement of various stent types," The American Journal of Cardiology, vol. 87, no. 1, pp. 34-39, 2001.

[6] C. J. Vrints, M. J. Claeys, J. Bosmans, V. Conraads, and J. P. Snoeck, "Effect of stenting on coronary flow velocity reserve: comparison of coil and tubular stents," Heart, vol. 82, no. 4, pp. 465-470, 1999.

[7] A. R. Williams, B.-K. Koo, T. J. Gundert, P. J. Fitzgerald, and J. F. LaDisa, "Local hemodynamic changes caused by main branch stent implantation and subsequent virtual side branch balloon angioplasty in a representative coronary bifurcation," Journal of Applied Physiology, vol. 109, no. 2, pp. 532-540, 2010.

[8] J. M. Garasic, E. R. Edelman, J. C. Squire, P. Seifert, M. S. Williams, and C. Rogers, "Stent and artery geometry determine intimal thickening independent of arterial injury," Circulation, vol. 101, no. 7, pp. 812-818, 2000.

[9] C. Rogers and E. R. Edelman, "Endovascular stent design dictates experimental restenosis and thrombosis," Circulation, vol. 91, no. 12, pp. 2995-3001, 1995.

[10] L. D. J. Jr, L. E. Olson, R. C. Molthen et al., “Alterations in wall shear stress predict sites of neointimal hyperplasia after stent implantation in rabbit iliac arteries," Ajp Heart \& Circulatory Physiology, vol. 288, no. 5, pp. H2465-H2475, 2005.

[11] R. S. Schwartz, K. C. Huber, J. G. Murphy et al., "Restenosis and the proportional neointimal response to coronary artery injury: results in a porcine model," Journal of the American College of Cardiology, vol. 19, no. 2, pp. 267-274, 1992.

[12] C. Chiastra, S. Morlacchi, D. Gallo et al., "Computational fluid dynamic simulations of image-based stented coronary 
bifurcation models," Journal of the Royal Society Interface, vol. 10, no. 84, p. 57, 2013.

[13] G. Liu, J. Wu, D. N. Ghista, W. Huang, and K. K. L. Wong, "Hemodynamic characterization of transient blood flow in right coronary arteries with varying curvature and sidebranch bifurcation angles," Computers in Biology and Medicine, vol. 64, pp. 117-126, 2015.

[14] E. Regar, G. Sianos, and P. W. Serruys, "Stent development and local drug delivery," British Medical Bulletin, vol. 59, no. 1, pp. 227-248, 2001.

[15] M. Gyöngyösi, P. Yang, A. Khorsand, and D. Glogar, "Longitudinal straightening effect of stents is an additional predictor for major adverse cardiac events," Journal of the American College of Cardiology, vol. 35, no. 6, pp. 1580-1589, 2000.

[16] J. J. Wentzel, M. D. Whelan, W. J. van der Giessen et al., "Coronary stent implantation changes 3-D vessel geometry and 3-D shear stress distribution," Journal of Biomechanics, vol. 33, no. 10, pp. 1287-1295, 2000.

[17] J. F. LaDisa, L. E. Olson, H. A. Douglas et al., "Alterations in regional vascular geometry produced by theoretical stent implantation influence distributions of wall shear stress: analysis of a curved coronary artery using 3D computational fluid dynamics modeling," Biomedical Engineering Online, vol. 5, no. 1, p. 1, 2006.

[18] A. M. Malek, S. L. Alper, and S. Izumo, "Hemodynamic shear stress and its role in atherosclerosis," Jama, vol. 282, no. 21, pp. 2035-2042, 1999.

[19] L. D. J. Jr, L. E. Olson, I. Guler et al., "Circumferential vascular deformation after stent implantation alters wall shear stress evaluated with time-dependent 3D computational fluid dynamics models," Journal of Applied Physiology, vol. 98, no. 3, pp. 947-957, 2005.

[20] J. F. LaDisa, L. E. Olson, I. Guler et al., "Stent design properties and deployment ratio influence indexes of wall shear stress: a three-dimensional computational fluid dynamics investigation within a normal artery," Journal of Applied Physiology, vol. 97, no. 1, pp. 424-430, 2004.

[21] X. Tian, A. Sun, X. Liu et al., "Influence of catheter insertion on the hemodynamic environment in coronary arteries," Medical Engineering \& Physics, vol. 38, no. 9, pp. 946-951, 2016.

[22] D. J. Patel and R. N. Vaishnav, Basic Hemodynamics and its Role in Disease Processes, pp. p1-504, University. Park Press, Baltimore, 1980.

[23] Z. Wang, A. Sun, Y. Fan, and X. Deng, "Comparative study of Newtonian and non-Newtonian simulations of drug transport in a model drug-eluting stent," Biorheology, vol. 49, no. 4, pp. 249-259, 2012.

[24] R. F. Wilson, D. E. Laughlin, P. H. Ackell et al., "Transluminal, subselective measurement of coronary artery blood flow velocity and vasodilator reserve in man," Circulation, vol. 72, no. 1, pp. 82-92, 1985.

[25] T. Chaichana, Z. Sun, and J. Jewkes, "Computation of hemodynamics in the left coronary artery with variable angulations," Journal of Biomechanics, vol. 44, no. 10, pp. 1869-1878, 2011.

[26] M. De Beule, P. Mortier, S. G. Carlier, B. Verhegghe, R. Van Impe, and P. Verdonck, "Realistic finite element-based stent design: the impact of balloon folding," Journal of Biomechanics, vol. 41, no. 2, pp. 383-389, 2008.

[27] J. Murphy and F. Boyle, "Predicting neointimal hyperplasia in stented arteries using time-dependant computational fluid dynamics: a review," Computers in Biology and Medicine, vol. 40, no. 4, pp. 408-418, 2010.

[28] W. Wu, W.-Q. Wang, D.-Z. Yang, and M. Qi, "Stent expansion in curved vessel and their interactions: a finite element analysis," Journal of Biomechanics, vol. 40, no. 11, pp. 2580-2585, 2007.

[29] C. G. Caro, J. M. Fitz-Gerald, and R. C. Schroter, "Atheroma and arterial wall shear. Observation, correlation and proposal of a shear dependent mass transfer mechanism for atherogenesis," Proceedings of the Royal Society of London. Series B, Biological Sciences, vol. 177, no. 177, pp. 109-159, 1971.

[30] C. K. Zarins, D. P. Giddens, B. K. Bharadvaj, V. S. Sottiurai, R. F. Mabon, and S. Glagov, "Carotid bifurcation atherosclerosis. Quantitative correlation of plaque localization with flow velocity profiles and wall shear stress," Circulation Research, vol. 53, no. 4, pp. 502-514, 1983.

[31] D. N. Ku, D. P. Giddens, C. K. Zarins, and S. Glagov, "Pulsatile flow and atherosclerosis in the human carotid bifurcation. Positive correlation between plaque location and low oscillating shear stress," Arteriosclerosis: An Official Journal of the American Heart Association, Inc.vol. 5, no. 3, pp. 293-302, 1985.

[32] B. Su, Y. Huo, G. S. Kassab et al., "Numerical investigation of blood flow in three-dimensional porcine left anterior descending artery with various stenoses," Computers in Biology and Medicine, vol. 47, no. 1, pp. 130-138, 2014.

[33] J. P. Archie Jr., S. Hyun, C. Kleinstreuer et al., "Hemodynamic parameters and early intimal thickening in branching blood vessels," Critical Reviews ${ }^{\mathrm{TM}}$ in Biomedical Engineering, vol. 29, no. 1, 2001.

[34] A. Colombo, E. Bramucci, S. Saccà et al., "Randomized study of the crush technique versus provisional side-branch stenting in true coronary bifurcations," Circulation, vol. 119, no. 1, pp. 71-78, 2009.

[35] Q. Liu and H.-C. Han, "Mechanical buckling of artery under pulsatile pressure," Journal of Biomechanics, vol. 45, no. 7, pp. 1192-1198, 2012.

[36] E. Cutrì, P. Zunino, S. Morlacchi, C. Chiastra, and F. Migliavacca, "Drug delivery patterns for different stenting techniques in coronary bifurcations: a comparative computational study," Biomechanics and Modeling in Mechanobiology, vol. 12, no. 4, pp. 657-669, 2013.

[37] A. Sun, Z. Wang, Z. Fan et al., "Influence of proximal drug eluting stent (DES) on distal bare metal stent (BMS) in multistent implantation strategies in coronary arteries," Medical Engineering \& Physics, vol. 37, no. 9, pp. 840-844, 2015. 